\title{
Automatically Moving Robot Intended for the Elderly with Voice Control
}

\author{
https://doi.org/10.3991/ijoe.v17i06.22299
}

\author{
Manussawee Piyaneeranart, Mahasak Ketcham ${ }^{(\varpi)}$ \\ King Mongkut's University of Technology North Bangkok, \\ Bangkok, Thailand \\ mahasak.k@itd.kmutnb.ac.th
}

\begin{abstract}
This research aims at an automatically moving robot intended for the elderly with voice control using voice recognition that gives a direction to the robot by a voice command. The development of this robot employed an algorithm that converts voice to text to control the robot mobility. The performance testing of the automatically moving robot intended for the elderly with Thai language voice commands revealed an accuracy of voice-to-text conversion for commanding the robot to move in designated directions of 100 percent, and the accuracy of voice-to-text conversion for commanding the robot to move in designated directions using English language was 80 percent, while that for both Thai and English commands was 70 percent. Errors in voice-to-text conversion occurred when commands were not clearly spoken or pronounced or when commands were issued from people located in a very noisy place.
\end{abstract}

Keywords-Automatically wheelchair, elderly, voice control

\section{Introduction}

Most elderly have encountered problems associated with medical services due to traveling expenses, medical expenses, and long waiting times for medical treatment. Moreover, buildings, equipment, or media do not support their access needs and utilization. Ultimately, these situations leave them abandoned and treated inappropriately since they are unable to access rehabilitation, and thus fundamental rights due elderly are violated. If elderly receive medical treatment and aiding equipment, they will be able to live their lives normally and do not become a burden to their families and the country's economy. Though the universal health care coverage scheme is available, granting of benefits, management, and mechanisms in service payment according to their rights are practically different, especially concerning assistive devices for the elderly. For example, a wheelchair that can assist the elderly, including the elderly with disabilities and the elderly with semidisabilities, has been designed to meet the needs of patients [1]-[4]. The adopted technology has the wheelchair to automatically [5]-[7] move in a safe position, and sensor signals are used to make driving easier [8], [9]. These technologies enable efficient wheelchairs to move in crowded places [10]. Such wheelchairs determine the position of movement within the building using 3D 
simulations to simulate the movement situation for the elderly to practice using wheelchairs effectively [11]-[14]. SLAM technology was used in the movement of the wheelchair [15], enabling the wheelchair to move outdoors by learning a large map and learning about urban features, such as pavement surfaces [16], [17] ramps or obstructions along the way [18]. In addition, wheelchairs have been improved to be more stable and able to move on uneven floors to prevent falls [19]. Wheelchairs are designed to be both sitting and standing, and the user can control wheelchairs by upper body movement [20]-[22]. Based on the abovementioned technology, current technology uses brainwave signals to control wheelchairs (electroencephalography: EEG) [23]-[27], hand gestures [28], [29], and images of the eye [30]-[32]. In addition, a network system has been installed on the wheelchair so that wheelchairs can communicate with each other to record the movement path. The movement of the wheelchair can be controlled in response to user guidance, and incidents of wheelchair users can be reported [33]-[35]. As a consequence, to reduce the gap of inaccessibility of healthcare services and aiding equipment as mentioned earlier, management and mechanisms of service payment need to be developed to reach a standard and eliminate such inequalities, including promoting operational guidelines for personnel and hospitals that will not be obstacles for elderly to access health care services any longer and perspectives [36] and attitudes of the management and people toward establishing a welfare state that has differences in terms of the historical context and social environment associated with the welfare of elderly. Some societies view that establishing good welfare for the elderly is worth the investment, while some societies consider welfare for the elderly as services responding to basic ethical principles in accordance with human rights. Different perspectives and attitudes have an effect on the content and substance of policies regarding elderly.

Multiple research studies have found that the development of management and mechanisms of service payment for elderly in Thailand should refer and compare to the way countries execute good standards of service management for elderly in the international context and that such an approach can be a lesson for developing the service system management for elderly in Thailand. However, it is not a conclusion that welfare for the elderly in Thailand has to follow those countries since it needs to be considered in association with requirements and other factors.

This research proposed the development of an automatically moving robot intended for the elderly with voice control (see Fig.1) that does not require the assistance of hospital staff to reduce the burden and number of nursing staff, provides fast service, and enables the hospital to increasingly accommodate other services, especially in hospitals that have a high ratio of service receivers to service providers, which is consistent with the situation in many hospitals across the country. Furthermore, the algorithms used in the automatically moving smart robot intended for the elderly with voice control can also be used to control electrical devices to facilitate the elderly who can speak but unable to move, lead a more comfortable life. 


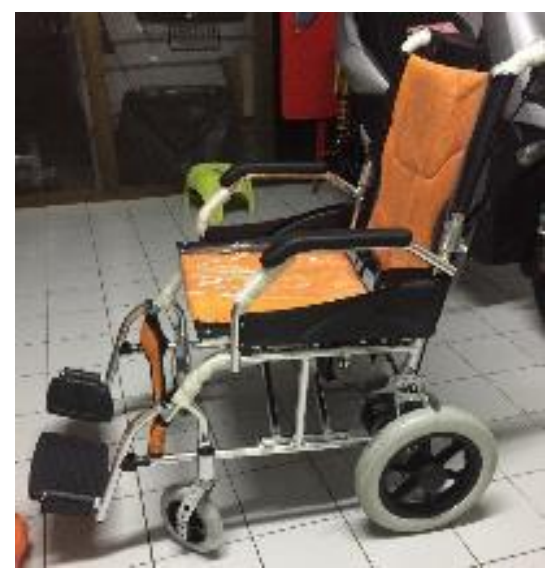

Fig. 1. A prototype wheelchair robot.

As shown in Fig. 1, fundamental structure of automatically moving robot intended for the elderly. The wheelchair used as the fundamental structure in this study has 4 wheels (the Quard) modified from a transport wheelchair that requires someone to push it.

\section{$2 \quad$ Literature Review}

\subsection{Wheelchairs}

Wheelchairs for patients and the elderly have evolved quickly with quality. Currently, wheelchairs for patients or the elderly use a lightweight metal structure such as aluminum or titanium. Electronic technology has been implemented in the control part by having a system to force and recline a backrest, a safety system and a system of fastmoving wheels suitable for elderly patients who need long-term wheelchair use. Thus, patients who require wheelchairs and patients with physical impairment caused by diseases or accidents can use wheelchairs to suit their individual needs in daily life.

\section{2 $\quad$ Sensors}

A sensor [37] is an object used to detect a situation or change of its own environment and provides a consistent outcome. A sensor is a transducer that produces different kinds of signals that are most likely electrical or light signals. Sensors are widely used in everyday life, such as a touch sensitive elevator button (touch sensor) and lamps with a dimmer touch base. In addition, sensors are used in countless situations that most people are unaware of. With the advancement of microelectromechanical systems and microcontroller platforms that are easy to use, the usability of sensors has been widely expanded rather than relying on conventional temperature, pressure, or flow 
measurement. Wireless sensors are also used in smart home systems [38] to detect temperature and control LED lamp.

\subsection{Microcontrollers}

Robots with higher conditions of function require an increase of robotic computer capabilities. Thus, a microcontroller has been invented to replace an electronic circuit. As such a fundamental system, a microcontroller can easily change operating conditions by changing the sequence control program run on personal computers. To build robot brains, robot operating control circuits and single board computers (SBCs) are implemented as controllers with an operating system similar to personal computers; however, everything is minimized and included in a small circuit that is popularly used in robots with multiple functions or complicated control systems, such as in programmable logic controllers (PLC). Programmable logic controllers were invented and developed to replace the relay automatic control system and are widely used in factories since they are durable enough to be used in a factory environment. Moreover, they are easy to use, have operating systems that can be changed easily, serve multipurpose needs and are easy for maintenance.

\subsection{Motor control}

Motor control means the ability to regulate a motor to operate as commanded to achieve safety of motors themselves and the machine equipment connecting to motors to achieve operator safety. The objectives of motor control are as follows: 1) starting motors, 2) stopping motors, 3) reversing, 4) motor running, 5) speed control, 6) safety of operators, 7) motor and system damage protection, and 8) maintenance of motor starting equipment

\subsection{Automatic speech recognition}

A keyboard and mouse were previously used as response devices for computers, including the ability to understand operating commands of computer systems, consequently contributing to access of technology that is difficult for many people. However, the arrival of automatic speech recognition (ASR) [39] destroyed the wall of technology, which made using computers so simple that people at all levels, though they are not familiar with and skillful in using technology, could respond to computer systems easily through voice. Voice or speech recognition is a system that helps convert voice to text in which computer systems receive voice data from electronic devices and choose appropriate language models for conversion [40].

\subsection{Basic principle of voice recognition}

This principle is similar to the basic of understanding speech or words spoken by any human that comprises sound wave analysis, a recognition decoder, a sound wave 
model, a dictionary, and a language model (rules and grammar). Voice conversions use a standard signal and writing system to comply with voice signals [41], [42].

\subsection{Language model, rules, and grammar}

Rules and grammar are used to identify methods of speaking that have a limited scope in voice conversion in voice recognition. A language model can facilitate analysis of speaking that does not have a definite form. Voice conversion is not comparable to a model of speaking, such as in the application of rules and grammar, but voice is converted into speech or words by means of the probability of words that are formed to be a sentence. Voice recognition using a language model to convert all speech or words into text is called dictation (the act of speaking words that someone writes down or that a machine records). The application of voice recognition to dictation can be used for voice conversion to text in a word processing program such as Microsoft Word as well as in other aspects, such as systems for flight reservations over the telephone, which can facilitate various speaking models and are more flexible and natural; moreover, an API system and AI technology can recognize voices and process spoken language as well [43].

\subsection{Relevant studies}

Hinderer et al. [44] proposed an autonomous stair-climbing wheelchair technology. It offers unlimited and independent mobility. Because of dynamic stabilization, the wheelchair moves on only one axis. It has highly agile driving behavior and is compact in size. The autonomous climbing system is based on a leg mechanism that consists of two legs with lower and upper leg support. While climbing a stairway, the legs push the wheelchair on the next higher situated step. Afterward, the legs are pulled successively on to the next step while climbing down, and the opposite sequence of movements occurs.

The work of Hashizume et al. [45] is discussed as follows. When the number of elderly people in a society increases, caregivers are increasingly needed. To reduce the burden of caregivers, a remote controllable electric wheelchair (telewheelchair) was developed. The telewheelchair is equipped with a remote-control function and a computational operation assistance function by means of a head mounted display (HMD). The caregiver does not need to constantly supervise the wheelchair user. In the meantime, the psychological burden faced by a person riding in a wheelchair is reduced if the wheelchair is operated by a machine without the caregiver's help. Technologies such as modern environmental sensors and artificial intelligence (AI) were used. The telewheelchair combines human operation with automatic operation using AI. AI performs object recognition and environment recognition and helps humans manipulate basic wheelchairs. In addition, the telewheelchair is operable from a remote location. This eliminates the need for a caregiver to physically move a heavy wheelchair, reducing the burden of the caregiver.

In the work of Josephine Leela et al. [46], consideration was made for those who encounter physical problems with walking due to injuries or disabilities, such that a 
wheelchair is brought to help disabled people take part in society and earn a living. Disabled people provide their voice to an Android mobile device; the output of the android mobile device is a voice command that is converted into text. The output of the mobile device is given to the microcontroller and the proposed system movement is controlled using a Bluetooth module. In addition, an ultrasonic sensor is used to detect obstacles.

In the work of Aktar et al. [47], a voice recognition-based intelligent wheelchair and GPS tracking system was developed for physically handicapped people who are unable to drive a wheelchair by hand so that they can operate the wheelchair using voice commands, and the location of patients/handicapped people can be traced using a GPS module in the wheelchair that tracks and sends the information to a smartphone application via Firebase. Voice module V3 is used to record the patient's voice and recognize that voice to follow the instructions of the patient. The patient's voice data are transmitted to a Wi-Fi module to control the wheelchair. The Wi-Fi module directs the motor driver to move the wheels to desired directions.

Avutu et al. [48] - As the number of the elderly and handicapped people encountering road accidents rises, the number of wheelchairs is expected to increase. However, the number of patients is greater than caregivers. As a consequence, a voice control module for a motorized wheelchair which works based on the speech processing technique and low cost local-map navigation and the concept of mel-frequency cepstrum coefficients (MFCCs) were employed so that the wheelchair will be automatically taken to desired destinations.

Barbosa et al. [49] proposed TrailCare, an indoor and outdoor context-aware system. TrailCare uses indoor and outdoor location information to assist wheelchair users. The outdoor system uses a GPS tracking system to record their trails, and the indoor system records the routes that the wheelchair passed to locations where an RFID card is installed that sends the position to the server. The TrailCare reader mounted on the wheelchair is Arduino middleware that has 3 features for communication, such as 1) communication through Bluetooth, 2) reading an RFID card to obtain the position information inside of the building, and 3) communication with the wheelchair using firmware software through RS-232.

Puanhvuan et al. [50] proposed a navigation-synchronized multimodal controlled wheelchair from brain signals (a brain-controlled wheelchair, BCW, that allows patients to control the wheelchair by their thoughts). P300 is a reliable brain electrical signal that could be used for interpreting user commands. Wheelchair users are able to select from 9 possible destination commands in the automatic mode and from 4 directional commands, such as forward, backward, turn left, and turn right.

Kundu et al. [51] proposed a hand gesture-based control of an omnidirectional wheelchair using an inertial measurement unit (IMU) and myoelectric units as wearable sensors. Seven common gestures are recognized to control the wheelchair, i.e., 1) forward, 2) clockwise, 3) turn left, 4) backward, 5) anticlockwise and 6) turn right. The gestures are classified using shape-based feature extraction and a dendogram support vector machine (DSVM) classifier. The dynamic gestures are mapped to the omnidirectional motion command to navigate the wheelchair. A single IMU is used to measure the wrist tilt angle and acceleration in 3 axes. EMG signals are extracted from 2 forearm 
muscles and processed to provide an RMS signal. Initiation and termination of dynamic activities are based on autonomous identification of the static to dynamic or dynamic to static transition by setting static thresholds on processed IMU and myoelectric sensor data. Classification involves recognizing the activity pattern based on the periodic shape of trajectories of the triaxial wrist tilt angle and EMC-RMS from the two selected muscles. Dynamic activities are used as features to classify dynamic activities. The classification algorithm and real-time navigation of the wheelchair use the proposed algorithm.

Lu'is Coelho and Daniela Braga [52] developed a voice-operated intelligent wheelchair using 41 commands. The principle of voice recognition used hidden Markov models (HMMS) to compare voice commands from users to the recorded commands. Other than the voice commands, this research study measured status values of users such as weight, vital signs, oxygen saturation, etc.

Shaheen and Umamakeswari [53] developed an intelligent wheelchair for people with disabilities by embedding a device to facilitate people with leg disabilities who cannot speak. Based on the ARM processor, the device is designed with an intelligent wheelchair, and people with disabilities can direct it. By pressing the keypad (buttons) in the device, disabled people can move the wheelchair. An L298 motor driver is used with the help of an ultrasonic obstacle sensor to detect obstacles. An LCD shows the basic needs of people with disabilities that are already assigned in the buttons.

Siva Kumar and Sudhagar. [54] developed a stair climbing intelligent wheelchair controlled by voice, a joystick, and navigational intelligence. The ATMEGA $3128 \mathrm{mi}-$ crocontroller is used to trace and control, and a set of sensors, such as infrared sensors and ultrasonic sensors, is used to detect obstacles.

E. Yulianto et al. [55] proposed a wheelchair for people with paralysis of both arms and legs or Quadriplegia Patient. The patients cannot use a joystick-controlled wheelchair. The electric wheelchair for people with paralysis of the arms and legs is designed to be controlled by an EMG signal. EMG signals are produced by contractions of the muscles that the paraplegic can move, including the neck and face muscles. The increased EMG signal amplitude during muscle contraction is used as a stimulant for the wheelchair electric motor to move forward, backward, right turn, and left turn. The muscle contractions to the electric motor are transmitted wirelessly to reduce cable use.

In regard to the relevant studies mentioned above, wheelchairs were controlled by using buttons or navigation equipment. The navigation or voice controlled parts relied on the principle of voice recognition through a microphone mounted on the Arduino controller equipment. Therefore, the idea considered in this research is to develop a wireless voice-controlled wheelchair with a collaborative operating system between a smartphone application and a Raspberry Pi 3 Model B board that have a similar performance to a computer and a higher processing performance than an Arduino board. 


\section{Propose Method}

In this research, we proposed the development of a voice control algorithm for an automatically moving robot intended for the elderly. In this section, we have developed two sections: robot development and design and propose a voice control algorithm.

\subsection{Robot development and design}

The component of the automatically moving smart robot intended for the elderly is shown in Fig 2.

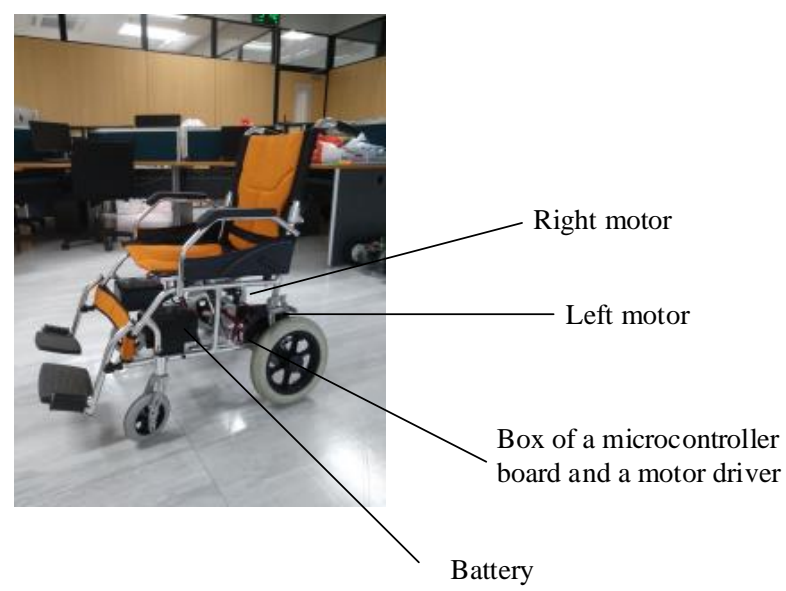

Fig. 2. The hardware component of the robot.

As shown in Fig.2, the hardware components of the robot, which are the components for controlling the robot. We have described the hardware components in the hardware component section.

Hardware components: for mobility control, a Raspberry Pi is implemented to perform processing. The devices used to control robot mobility consist of the Cytron 2x10A Motor Driver HAT, 2 units of a 12 volt, 12 amp battery, and 2 units of a 24 volt, 350 Watt gear motor, and Data transmission. The hardware system uses a Raspberry $\mathrm{Pi}$, a small computer board having efficient performance comparable to a small computer that can facilitate the display operation. The Ubuntu or Raspbian operating system can be installed, as seen in Fig 3a, and an SD card is used to store data, comparable to a computer hard disk, as seen in Fig $3 b$. 


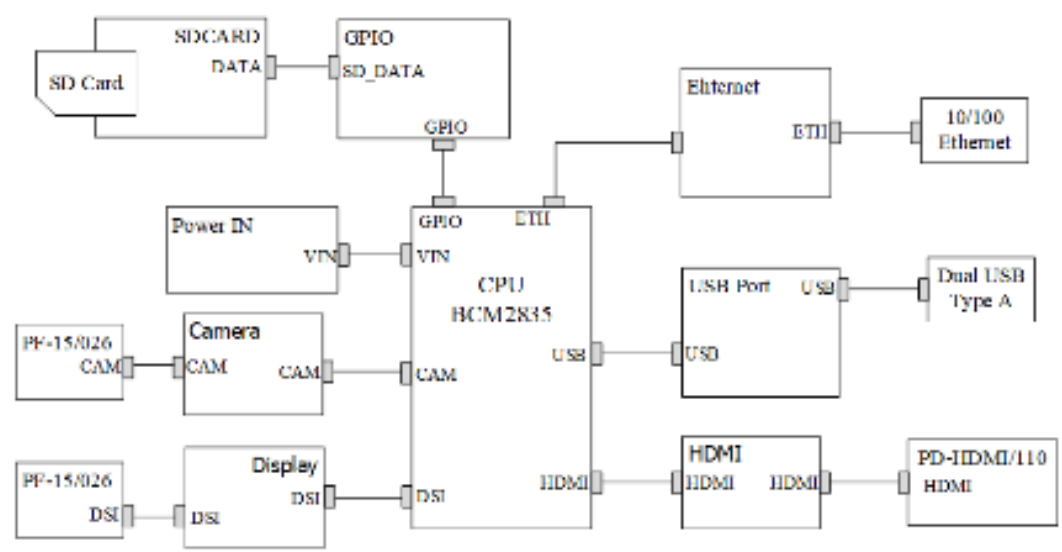

(a)

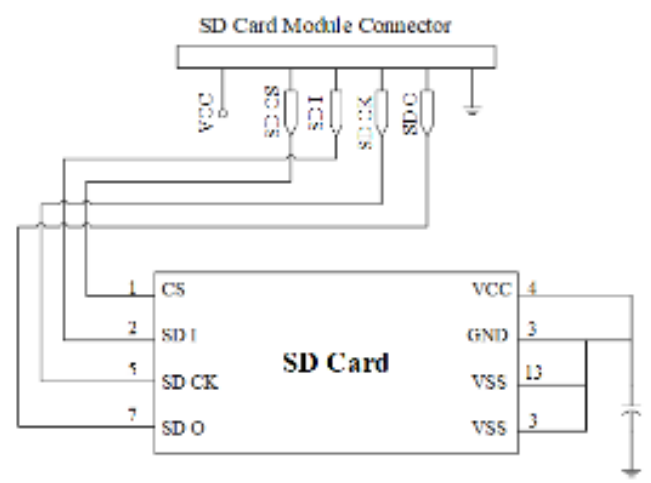

(b)

Fig. 3. Microcontroller Board and Micro SD Card.

As shown in Fig. 3, (a) the microcontroller board is analogous to the robot's brain, and the wheelchair works to process commands for the robot. (b) the SD card serves as a storage device.

The Cytron 2x10A motor driver HAT: This motor driver is a 2-channel DC motor drive that constantly facilitates a current of 10 amps (maximum 30 amps, not longer than 10 seconds) and 6-24 volts of direct current. The HAT board is designed to plug directly into the Raspberry Pi via a 40-pin connector to control direction. The specifications are as follows: MOTOR1=GPIO26, MOTOR2=GPIO24, and pulse-width modulation (PWM) controls the rotating speed from the microcontroller, while MOTOR1=GPIO12, MOTOR2=GPIO13, and the switch test and light showing motor rotation are available, as seen in Fig 4. 


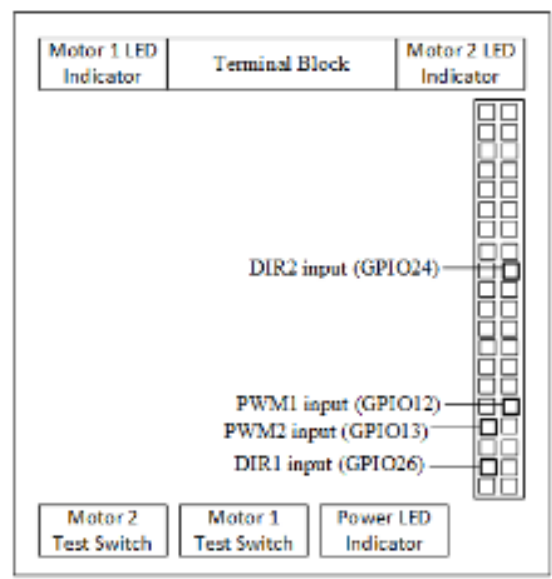

Fig. 4. Cytron 2x10A Motor Driver HAT.

The DC motor drive board controls the direction of the motor motion that is commanded by the control board.

12 volt, 12 amp battery: The 12 volt, 12 amp batteries, as seen in Fig 5a, are used to deliver electric current to the circuits and motors to work without connecting to power sockets, but the batteries will be connected to the robot system to deliver electric current to the microcontroller board, electric circuit, and motors, enabling the movement of the robot. In this study, 2 units of batteries were used to deliver electric current to the motors mounted on the left rear wheel and right rear wheel, as seen in Fig 5b.

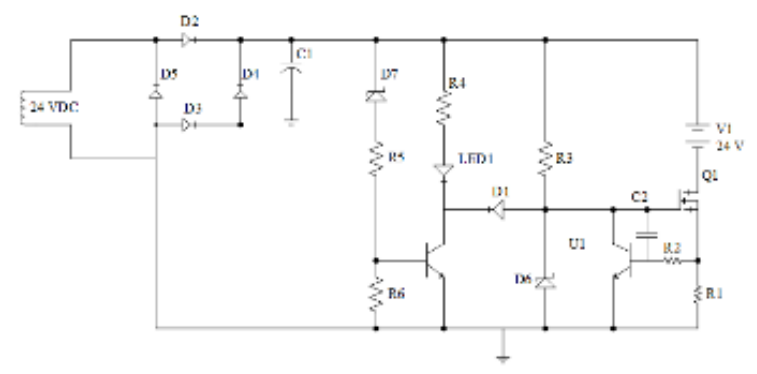

(a)

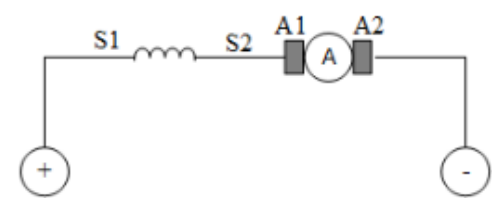

(b)

Fig. 5. Battery and Motor. 
Regarding the 24 volt and 350 Watt gear motor: when the motor control circuit delivers direct electric current to the motors as seen in Fig 6a, the motors will rotate according to the control kit controlled by facilitating two-directional rotation, namely, the clockwise direction and anticlockwise direction that facilitate the PWM system. The rotational speed adjustment is represented as a percentage according to commands of the motor control circuit. In this study, 2 units of motors mounted on the left rear wheel and right rear wheel were used, as seen in Fig 6 b.

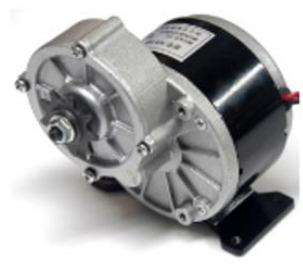

(a)

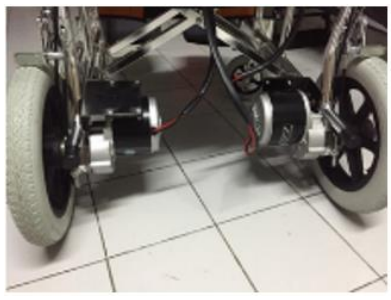

(b)

Fig. 6. Installation of the motor on the wheel.

As mentioned earlier, the hardware control of robot mobility was connected via electric circuits to control the mobility of the 2 motors that start from the direct connection of the motor control circuit to the microcontroller board, as seen in Fig 7a. In this study, the microcontroller board and DC motor driver board operating together with an Android smartphone application developed for voice commands were used to command the motors to work as required by using $12 \mathrm{~V}$ electricity to deliver electric current to the motors according to the circuit diagrams, as seen in Fig $7 \mathrm{~b}$.

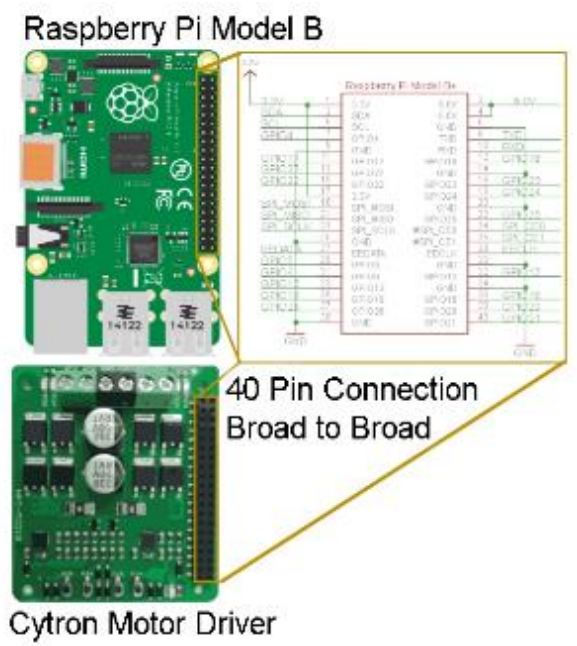

(a) 


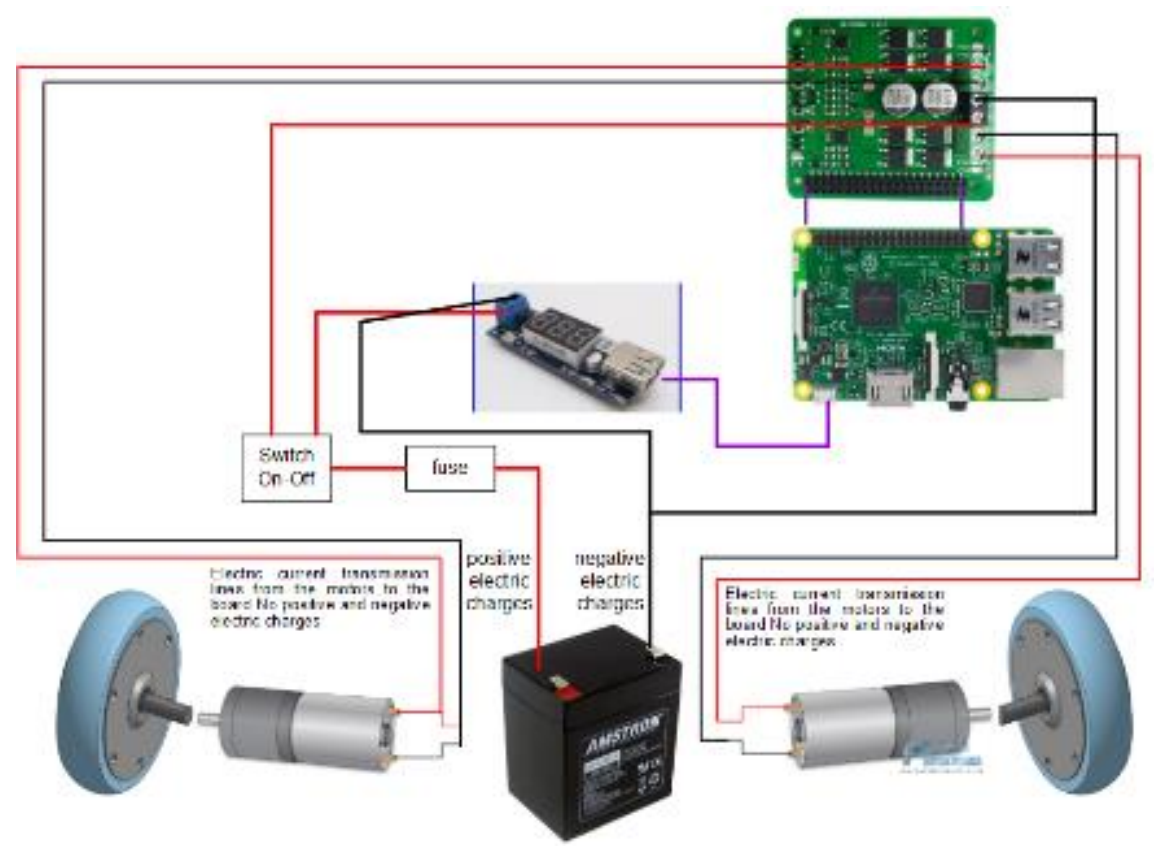

(b)

Fig. 7. Motor control circuit diagram.

Data transmission: the wheelchair and the input device will be connected to the signal Wi-Fi network. Wi-Fi is distributed from the input device, causing the input device to act as a router device. When the system installed in the wheelchair is opened, the system will connect to the client device via Wi-Fi network as shown in fig. 8 .

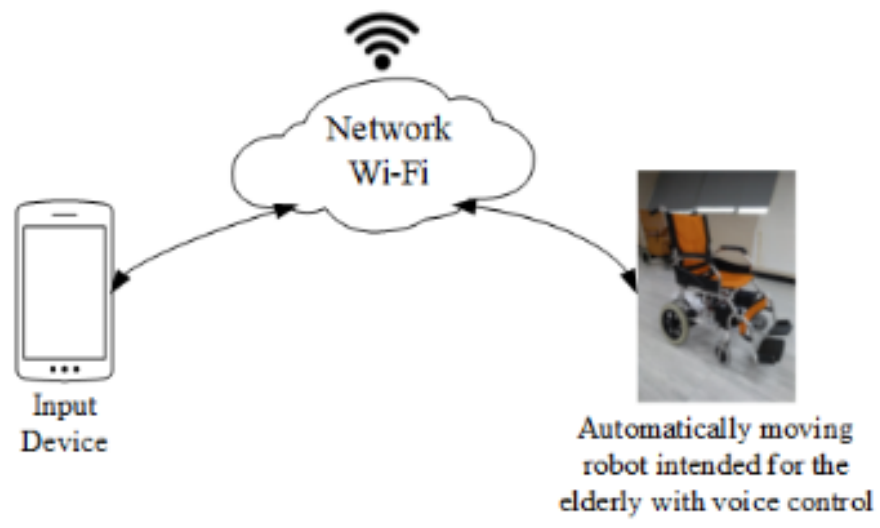

Fig. 8. Data transmission between the client device and an automatically moving robot intended for the elderly with voice control 
As shown in figure 8, the systems are connected together on the same Wi-Fi network. The microcontroller board receives voice commands from the input device to process and send the processed voice commands to control the movement of the robot.

Robot control System: this section describes the operation control systems for the wheelchair as follow voice command, processing, and robot control. Each motor is commanded to move in 2 directions, namely, forward and backward, independently of each other which can control the direction of mobility that determines the motor rotation, as shown in Fig 9a, and the operation diagram is shown in Fig 9b, while directions of motor mobility are shown in Table 1.

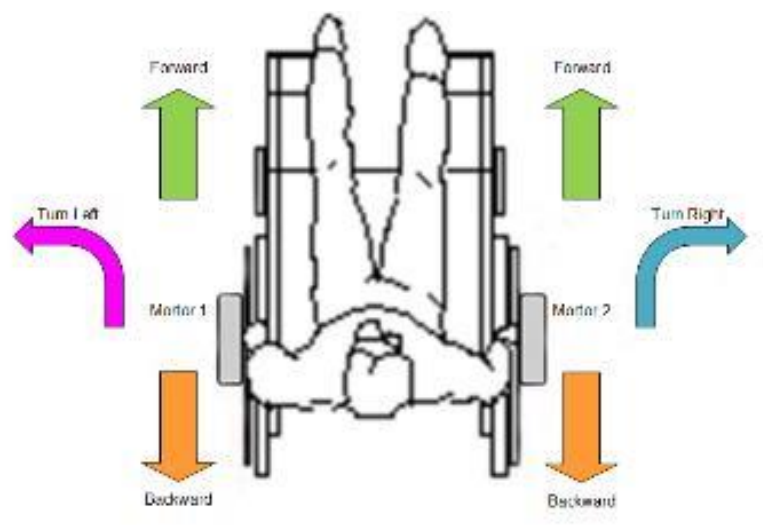

a) Directions of robot mobility

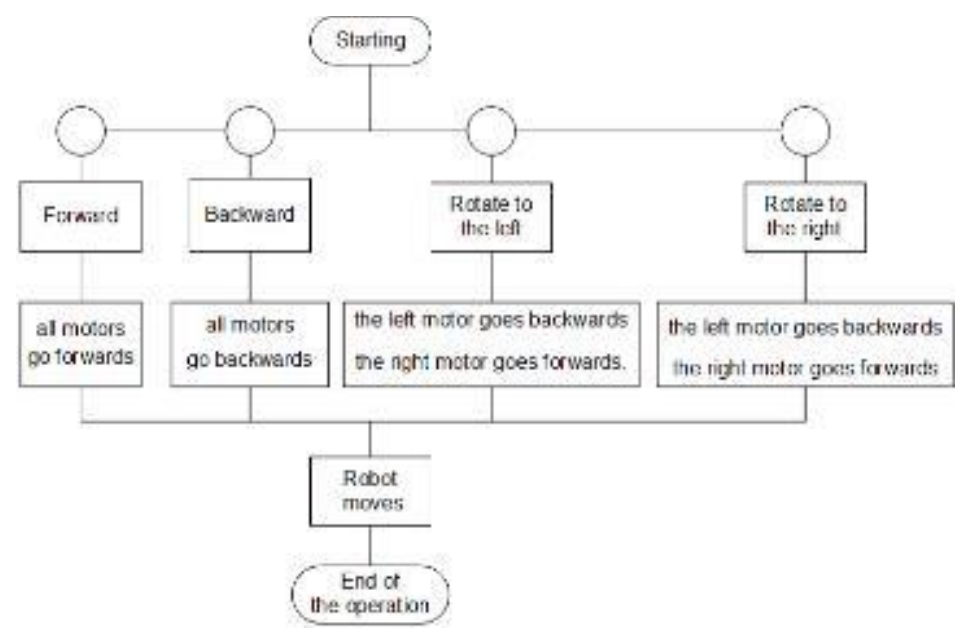

(b) Diagram of mobility control operation.

Fig. 9. A prototype wheelchair robot. 
As shown in Fig. 9, Fundamental structure of automatically moving robot intended for the elderly. (a) Directions of robot mobility. (b) Diagram of mobility control operation.

Table 1. Motor rotation in accordance with command direction.

\begin{tabular}{|l|c|c|c|c|}
\hline \multirow{2}{*}{ Direction } & \multicolumn{2}{c|}{ Go forward } & \multicolumn{2}{c|}{ Go backward } \\
\cline { 2 - 5 } & Motor 1 & Motor 2 & Motor 1 & Motor 2 \\
\hline Go Forward & 1 & 1 & 0 & 0 \\
\hline Go backward & 0 & 0 & 1 & 1 \\
\hline Turn left & 0 & 1 & 1 & 0 \\
\hline Turn right & 1 & 0 & 0 & 1 \\
\hline
\end{tabular}

The movement of the elderly wheelchair robot uses 2 motors to control each direction. The number 1 indicates that the motor is running and the number 0 indicates that the motor is not running. Go forward direction, motor 1 and motor 2 will rotate forward. Go backward direction, motor 1 and motor 2 will rotate backwards. Turn left direction, motor 1 will rotate backwards and motor 2 will rotate forward. Turn right direction, motor 1 rotates forward and motor 2 rotates backwards.

\subsection{Propose a voice control algorithm}

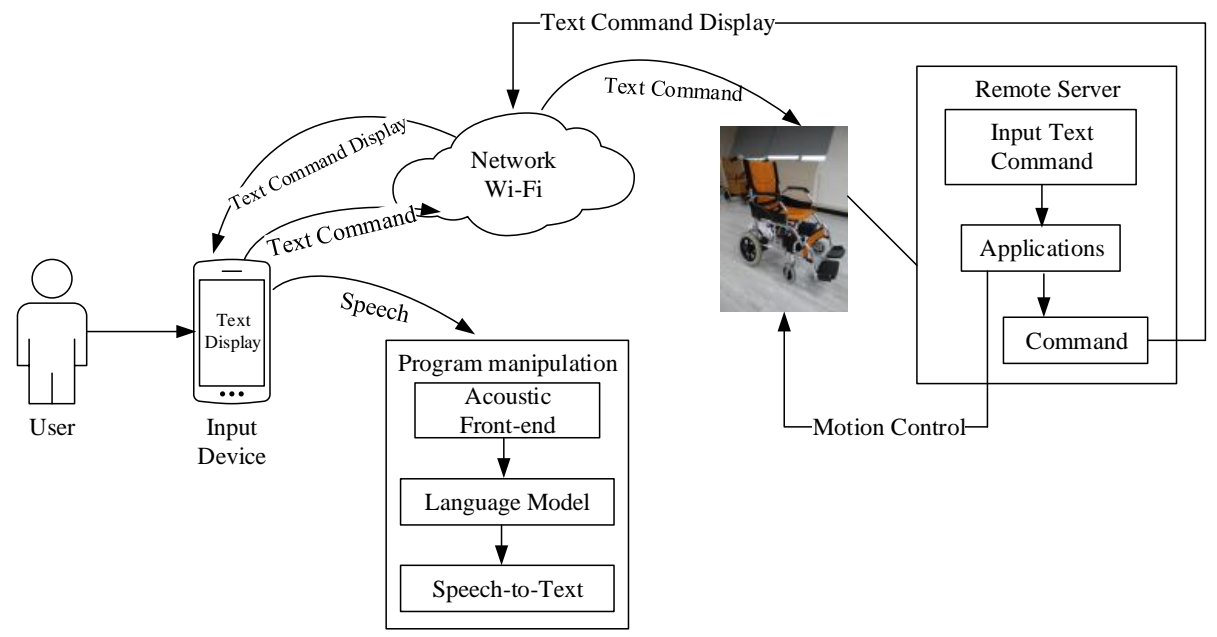

Fig. 10.The operation of devices and program used to receive data.

As shown in Fig.10, the equipment of the system refers to the communication between a person and a wheelchair robot. With communication, the person will use voice commands to control the wheelchair robot with a client device connected to the same Wi-Fi network. Data reception is operated by a microcontroller. The controller opens the system to connect to a robot using a Wi-Fi system and wireless client devices to receive voice commands. Next, the program shall use voice recognition for command 
classification and control the robot. A program manipulation is an operation within an input device where the client device receives speech signals from people and sent the command into the microcontroller via the same Wi-Fi network. The connection between the client device and the robot were connected via a Wi-Fi network. The control command that is sent to the microcontroller board is processed to be used to control the movement of the robot.

This study developed an algorithm to control an automatically moving robot intended for the elderly with voice control based on the procedures program manipulation and remote server.

Program manipulation: This section describes the operation of the system installed inside the input device. This is a system operation install into the input device, consisting of an Acoustic front-end, Language Model, and Speech-to-text procedure.

Acoustic front-end: When voice signal data were received, the voice signals were converted to frequencies to build a voice signal standard using a voice management system. The estimated power spectrum was calculated. For the analysis windows, windows were put in the scope of analysis, as seen in Fig. 11, by multiplying each value of the signals in a voice data frame by a function frame value, as shown by Eq (1):

$$
W(n)=0.54+0.46 \cos \left(\frac{2 \pi n}{N-1}\right)
$$

Where $W(n)$ is windows in time, $\mathrm{N}$ is the amount of data per frame. Next, signals employed the fast Fourier transform and the power of 2 (squared) and power spectrum were calculated according to $\mathrm{Eq}(2)$ :

$$
P(\omega)=\operatorname{Re}[S(\omega)]^{2}+\operatorname{Im}[S(\omega)]^{2}
$$

Where $P(\omega)$ is the power spectrum, $S(\omega)$ is the signal in the frequency domain that employed the Fourier transform.

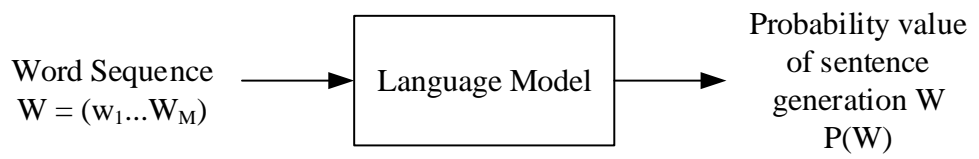

Fig. 11.Probability value of the sentence generation.

As shown in Fig. 10, the sentence probability is an approximation of the power spectrum for the analysis windows, which is done by placing the window in the region to be analysed.

Voice signals were built to be voice signal standards, and the standardized signals were transferred to the dictation system by analysing the voice energy value of each frame in a short period. Voice energy value of each point in a frame was calculated by Eq (3):

$$
E j=\sqrt{\left(\sum_{i=j}^{j+n} X_{i}^{2}\right) / n}
$$


where $E j$ is voice energy value at the point $j, X_{i}$ is the amplitude value of the voice signal at the point $\mathrm{i}$, and $\mathrm{n}$ is the number of points used in the calculation. The mean voice energy value of any frame is calculated by $\mathrm{Eq}(4)$ :

$$
E_{\text {avg }}(F)=\left(\sum_{j=F} E_{j}\right) /|F|
$$

where $F$ is a frame or set of points to be calculated.

Language model: The dictation system was implemented to generate messages consistent with the original speech. Messages were compared to designated commands for commanding the motors to operate. The motor operation occurs in the hardware part used to control the mobility of the automatically moving robot intended for the elderly, which includes 2 units of motors for the left rear wheel and right rear wheel of the wheelchair, gears, chains, 2 units of batteries for the motors controlling the left rear wheel and right rear wheel, and a fuse. These devices were installed on the wheelchair with 4 wheels (the Quard) and connected to the microcontroller board, as seen in Fig. 12.

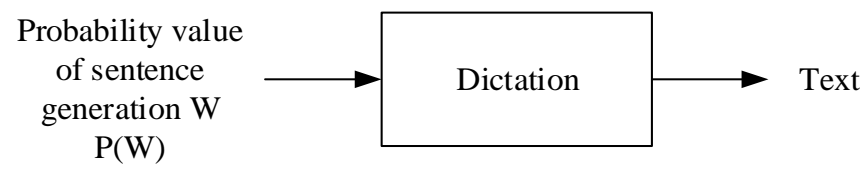

Fig. 12. Text creation.

The dictation system was implemented to generate messages consistent with the original speech. Messages were compared to designated commands for commanding the motors to operate.

Speech-to-text procedure: This is a step that received the vocabulary from the language model step and collects the vocabulary into text using Eq.5.

$$
\widehat{w}=\underset{w \in W}{\arg \max _{w} P(\mathrm{Z} \mid w) P(w)}
$$

whereZ is the observation sequence received from voice signals, $w$ is word sequence of voice signals, $W$ is the set of all possible word sequence received from the voice recognition, and $\widehat{w}$ is the set of word sequences received from the voice recognition.

Remote server: This section shows the operation of the system that installed on the wheelchair. It consists of 3 steps as Text Input, Applications, and Command.

Input text command: This section received voice commands from the input device that has been converted to text command. We defined command in two languages: Thai and English. There are commands to Go forward, Go backward, Turn left, and Turn right. Text commands have been converted to numeric commands representing forward, backward, left turn, and right commands to numbers 0001, 0010, 0011, and 0100 respectively. Then the system will send a command signal to the Application step to carry this command signal to control the GPIO, as follows: 


\section{Algorithm 1: Convert text command to number}

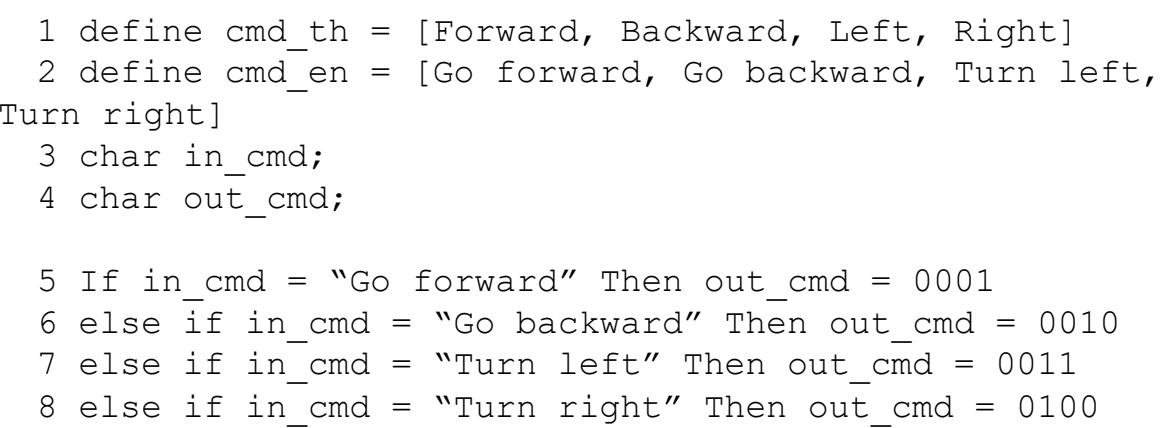

Applications: This procedure receives the command signal and controls the GPIO PIN. The GPIO PIN is sent to the motor drive to control the movement of the wheelchair, as follows:

\section{Algorithm 2: Control motor}

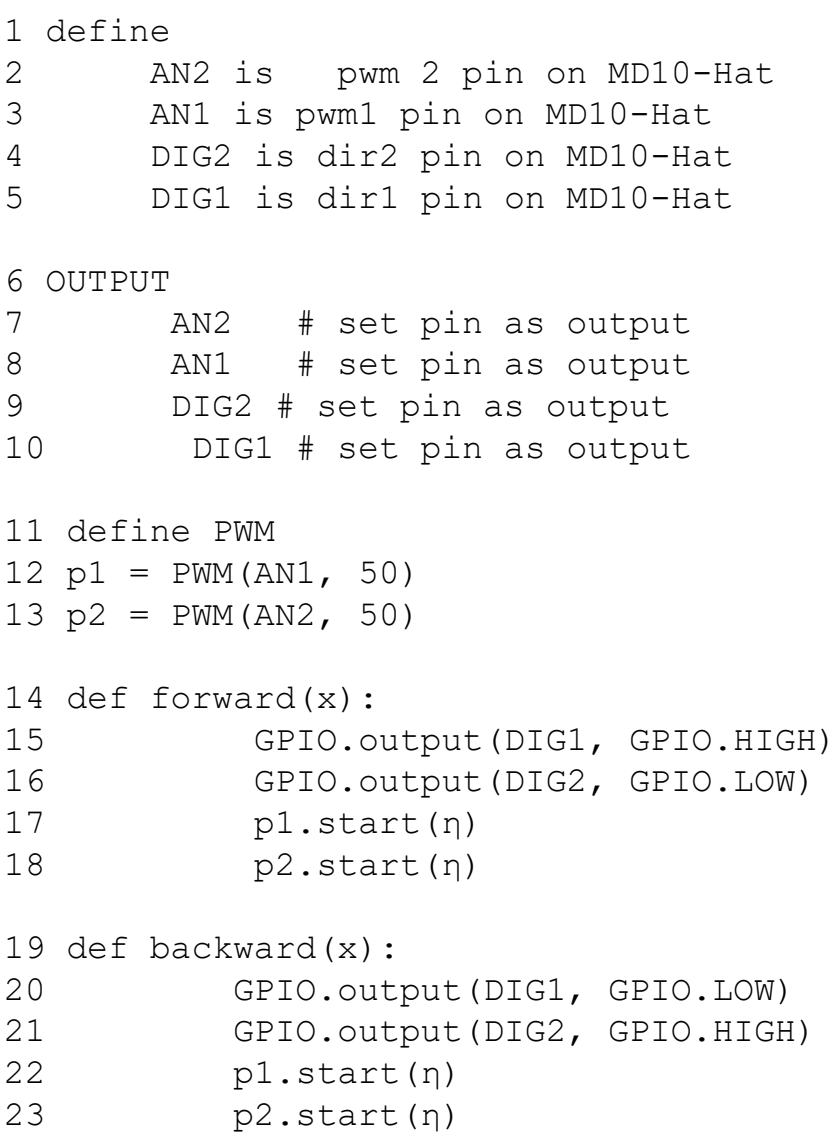




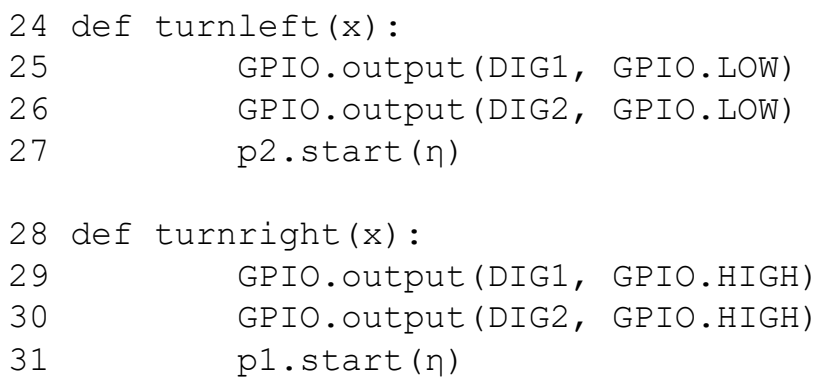

As algorithm 2 shows the value $\eta$, where $\eta$ represents the motor speed assigned to the wheelchair for use in motion.

Command: this step receives the GPIO PIN from the application step to control the motor of the wheelchair. The GPIO PIN value allows the wheelchair to move in the direction of the command it receives and displays the voice command as a text on the input device screen. There are steps to work as follows:

\section{Algorithm 3: Command display on the input device}

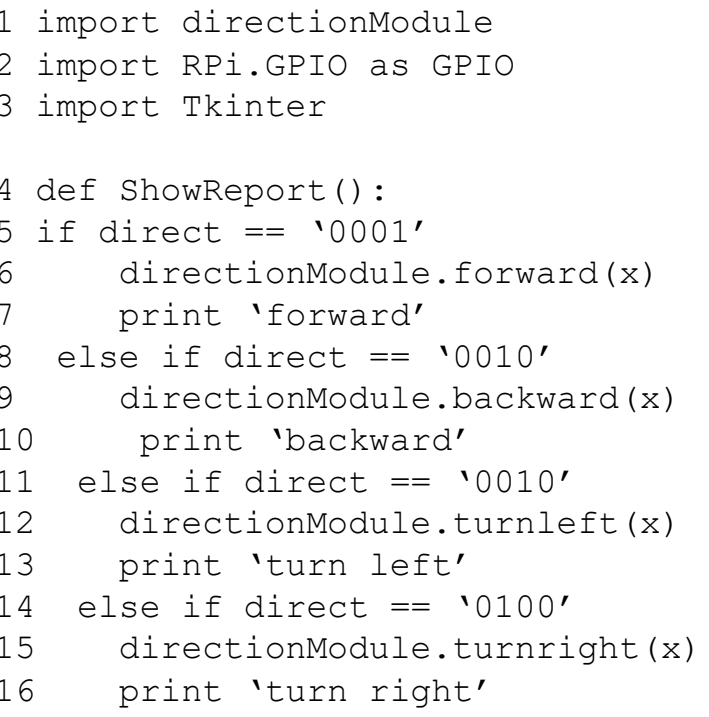

\subsection{Usability testing with the prototype}

Usability testing with the prototype of the automatically moving robot intended for the elderly with voice control includes the following procedures:

Test of connected devices. An Android phone is connected to a Raspberry PI Board and the motors controlling the left rear wheel and right rear wheel. All devices are commanded to work to check if they run normally or not.

Errors are corrected and testing of all operations is performed until everything runs correctly and completely. 


\subsection{Evaluation}

After performing the installation and test of the automatically moving robot prototype, the prototype performance is evaluated according to the following:

The results of developing the automatically moving robot intended for the elderly with voice control.

The results of converting voice signals to text messages.

\subsection{Data analysis}

The analysis of the data for accuracy in this document was used statistical analysis with an emphasis on the evaluation of the accuracy of voice commands. Accuracy analysis uses a method to verify the robot control with voice commands in Thai and English each time. It is calculated according to equation (6).

$$
\text { Accuracy }=\frac{n}{N} \times 100 \%
$$

where $n$ is the number of instructions that have performed correctly, $N$ is the total number of commands.

\section{$4 \quad$ Experiments and Results}

The results of designing and developing the automatically moving robot intended for the elderly with voice control comprise the following procedures:

The evaluation of the results of the development of the robot. The automatically moving robot intended for the elderly with voice control was designed and developed in 2 parts as the application for receiving voice signals and the control part of robot mobility, as seen in Fig 13a and 13b, respectively. The case of the speaking test with an application is shown in Fig 13c.

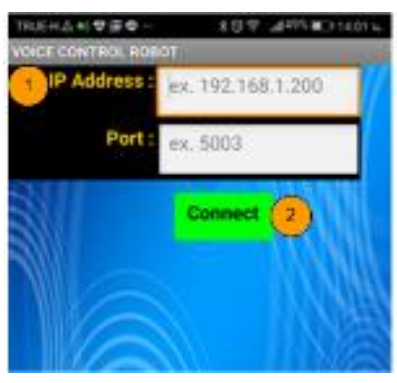

a)

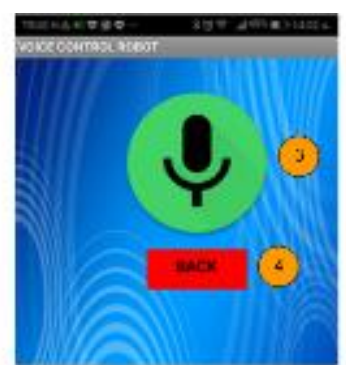

b)

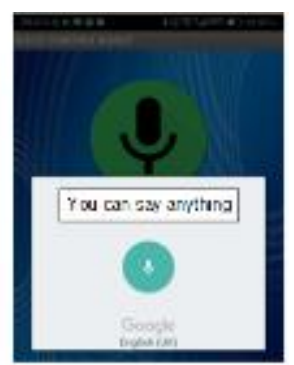

c)

Fig. 13. Voice control robot application. 
As shown in Fig. 13, (a) The application used for system connection, number 1 - Fill in IP address and port of the robot and number 2 - Buttons connected to voice command system. (b) the application used to receive voice commands, number 3 - Push button for voice command and number 4 - Back button to the system connection window. (c) Application used for receiving voice commands.

System operation: The automatically moving robot intended for the elderly with voice control through wireless smartphone devices was tested by speaking to the application, and it was found that if the voice command was spoken clearly and correctly, the robot was able to move well as per designated directions and voice commands, namely, go forward, go backward, turn left, and turn right. There are voice commands displayed in Thai and English as shown in Figures 14a and 14b, respectively.
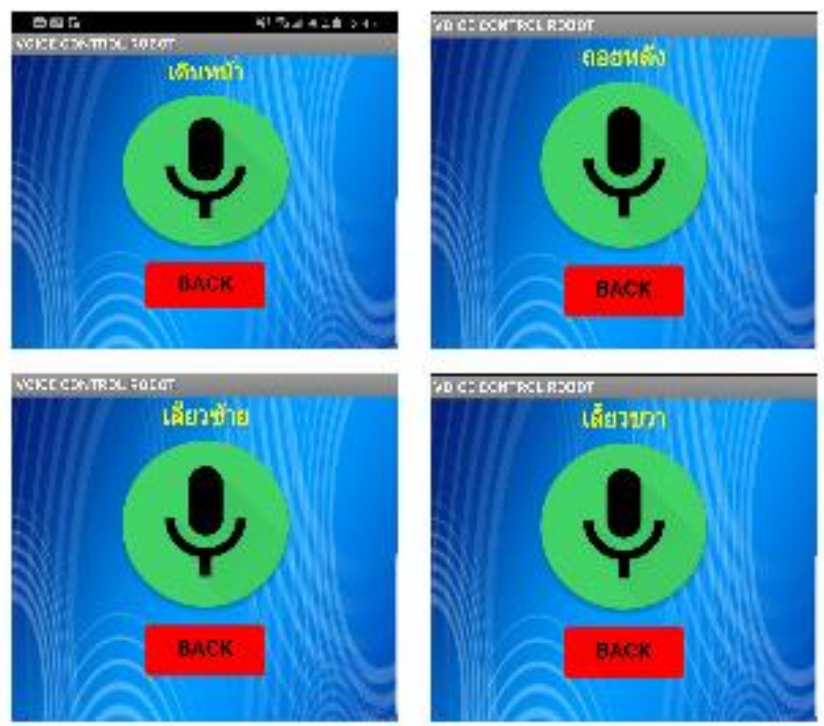

a) Display the results of Thai voice commands 

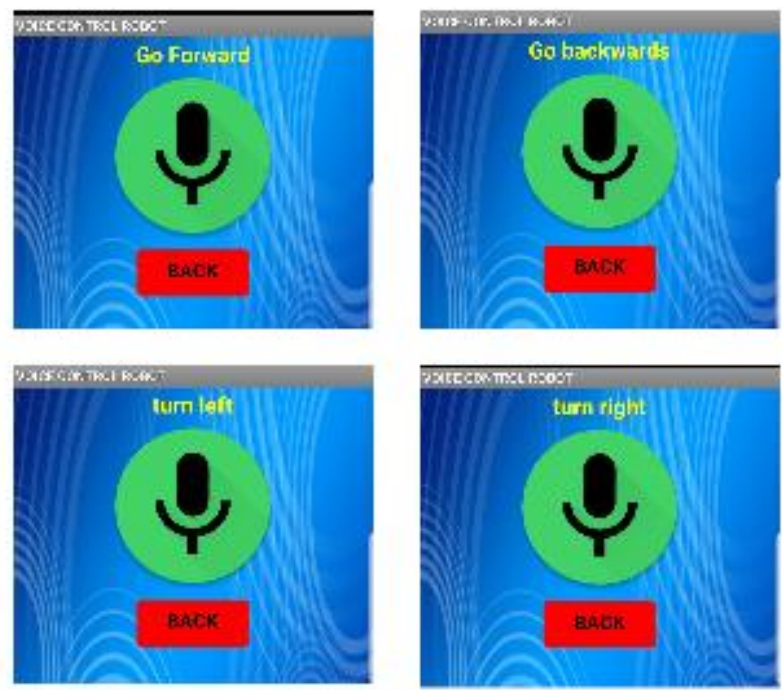

b) Displays the results of English voice commands

Fig. 14. Robot wheelchair prototype.

As shown in Fig. 14, (a) display the results of Thai voice commands and (b) displays the results of English voice commands.

\subsection{Experiment of robot mobility}

The experiment consists of evaluating the robot mobility through the application on wireless smartphone devices. The experiment of using voice commands to control the robot indicated that if voice commands were spoken correctly, the robot could move well according to the directions of go forward, go backward, turn left, and turn right, but if voice commands were unclearly spoken or disturbed by noise, errors could occur in the voice conversion. In addition, if the Wi-Fi signal was poor, signals communicating with the robot and the wireless smartphone devices were disconnected. The test results of robot mobility are shown in Figs 15-18. 


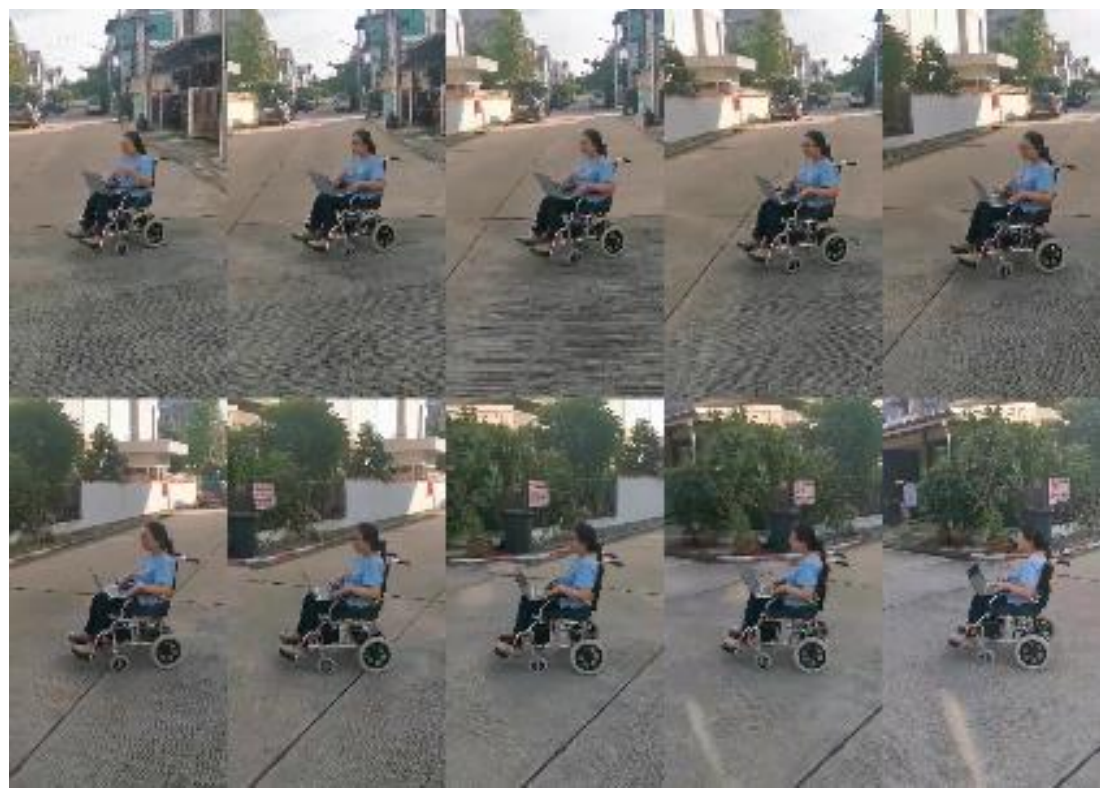

Fig. 15.The robot mobility with going forward.

As shown in Fig. 15, this is the experimental movement of the wheelchair robot for the elderly to move forward, which employs voice control.

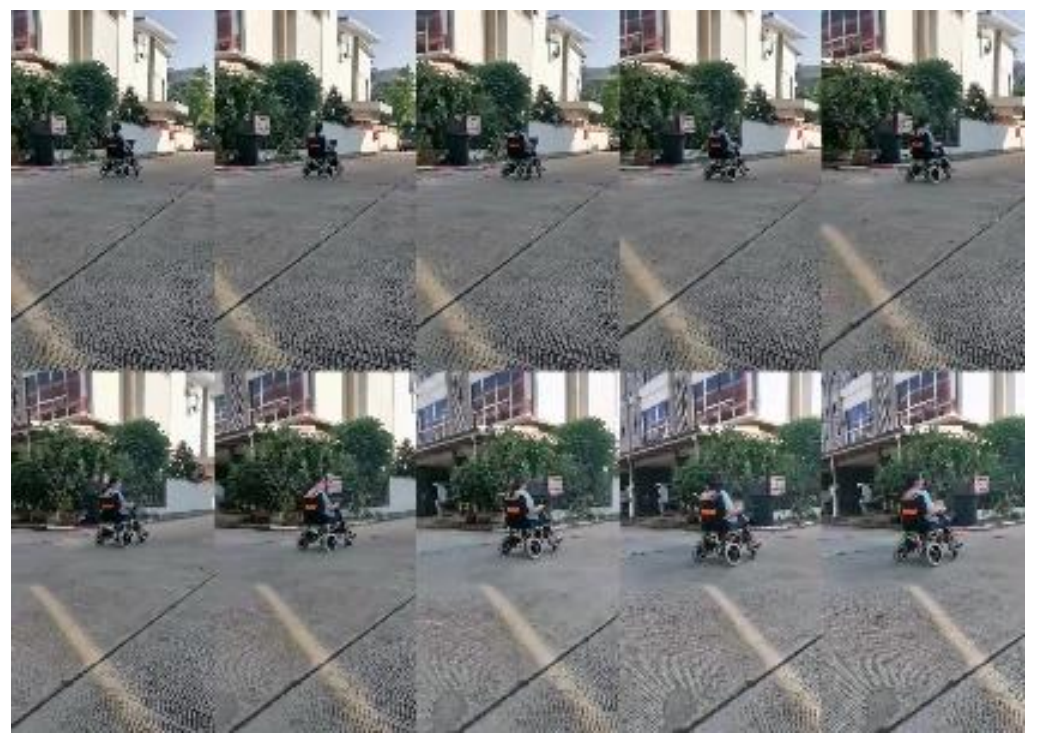

Fig. 16.The robot mobility with going backward. 
As shown in Fig. 16, the experimental movement of the wheelchair robot for the elderly to go backward.

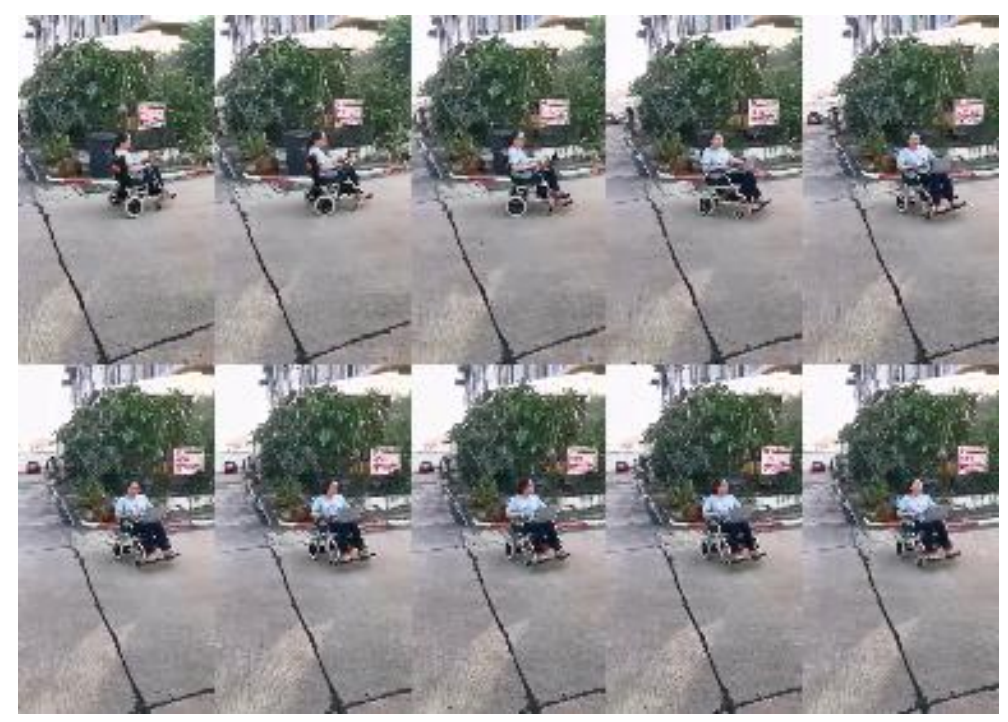

Fig. 17.The robot mobility with making a right turn.

As shown in Fig. 17, the experimental movement of the wheelchair robot for the elderly to turn right.

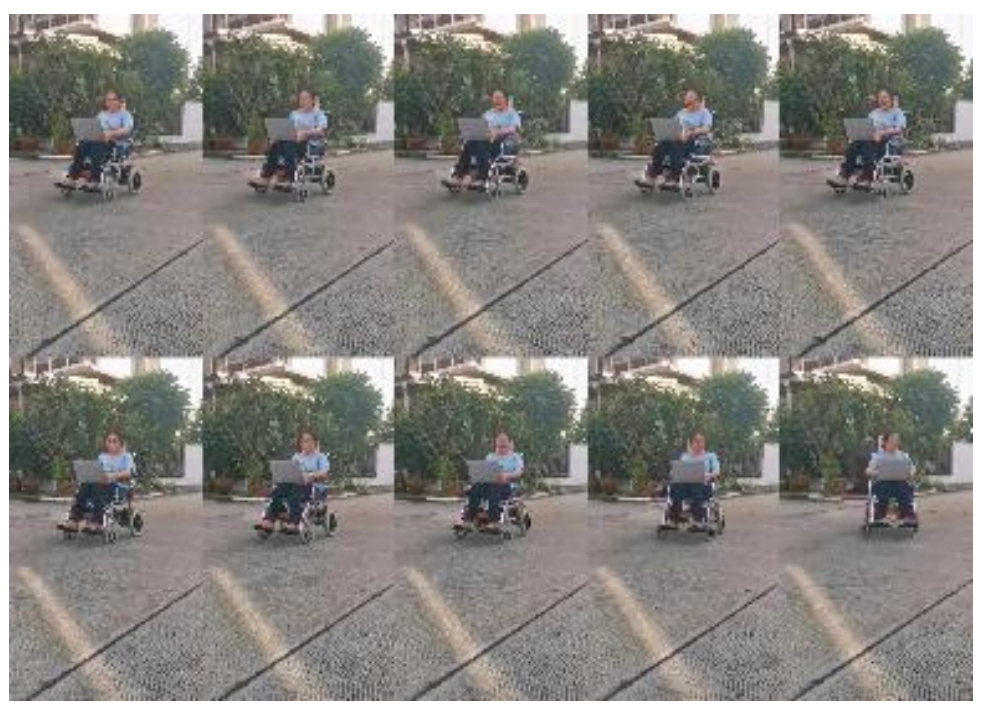

Fig. 18.The robot mobility with making a left turn. 
As shown in Fig. 18, the experimental movement of the wheelchair robot for the elderly to turn right.

\subsection{Evaluation results of voice-to-text conversion}

This study conducted a mobility experiment of the automatically moving robot intended for the elderly with voice control through wireless smartphone devices by allowing test participants to speak through the application on the wireless smartphone devices with 4 commands, such as go forward, go backward, turn left, and turn right based on 3 patterns of testing, namely, Thai language commands, English language commands, and Thai/English language alternating commands. The results are shown in Table 2.

Table 2. The set of voice commands.

\begin{tabular}{|l|c|c|c|}
\hline $\begin{array}{c}\text { Set of } \\
\text { voicecommands }\end{array}$ & $\begin{array}{c}\text { Test in Thai language(Go forward, } \\
\text { Go backward,Turn left, Turn right) }\end{array}$ & $\begin{array}{c}\text { Test in English } \\
\text { language(Forward, } \\
\text { Backward,Left, Rgiht) }\end{array}$ & $\begin{array}{c}\text { Thai/English(la } \\
\text { nguagealternati } \\
\text { ngcommands) }\end{array}$ \\
\hline Correct & 10 & 8 & 7 \\
\hline Incorrect & - & 2 & 3 \\
\hline Percent & 100 & 80 & 70 \\
\hline
\end{tabular}

Table 2 shows that, experiment with voice command set, including Thai voice, English voice and Thai/English language alternating commands. The Thai voice command set is 100\% accurate, the English voice command set is $80 \%$ correct, and Thai/English language alternating command set is $70 \%$ correct.

Table 2 shows 3 patterns of testing, i.e., 10 times of Thai language voice commands, 10 times of English language voice commands, and 10 times of Thai/English language alternating voice commands. The test results revealed the following:

By testing 10 times using Thai language voice commands, the system could correctly convert voice commands to text 10 times, making the robot move to the correct directions 10 times, as shown in Table 3.

By testing 10 times with English language voice commands, the system could correctly convert voice commands to text 8 times, making the robot move to the correct directions 8 times, and the system could not correctly convert voice commands to text 2 times, making the robot move to the wrong directions 2 times, as shown in Table 4.

By testing 10 times with Thai/English language alternating voice commands, the system could correctly convert voice commands to text 7 times, making the robot move to the correct directions 7 times, and the system could not correctly convert voice commands to text 3 times, making the robot move to the wrong directions 3 times, as shown in Table 5. 
Table 3. Mobility test result of the automatically moving robot intended for the elderly with Thai language voice commands.

\begin{tabular}{|c|l|l|c|}
\hline No. & \multicolumn{1}{|c|}{ Voice commands } & \multicolumn{1}{|c|}{ Voice commands to text conversion } & Correctness \\
\hline 1 & Forward & Forward & Y \\
\hline 2 & Backward & Backward & Y \\
\hline 3 & Left & Left & Y \\
\hline 4 & Right & Right & Y \\
\hline 5 & Backward & Backward & Y \\
\hline 6 & Forward & Forward & Y \\
\hline 7 & Left & Left & Y \\
\hline 8 & Left & Left & Y \\
\hline 9 & Stop & Stop & Y \\
\hline 10 & Right & Right & Y \\
\hline & & \multicolumn{1}{|c|}{ Percentage } & $100 \%$ \\
\hline
\end{tabular}

Table 4. Mobility test result of the automatically moving robot intended for the elderly with English language voice commands.

\begin{tabular}{|c|l|l|c|}
\hline No. & \multicolumn{1}{|c|}{ Voice commands } & \multicolumn{1}{|c|}{ Voice commands to text conversion } & Correctness \\
\hline 1 & Go forward & Go forward & Y \\
\hline 2 & Go back & Go backward & Y \\
\hline 3 & Turn left & Turn left & Y \\
\hline 4 & Turn right & Turn right & Y \\
\hline 5 & Back & Black & N \\
\hline 6 & Go forward & Go forward & Y \\
\hline 7 & Turn left & Turn left & Y \\
\hline 8 & Stop & Stop & Y \\
\hline 9 & Back & Black & N \\
\hline 10 & Turn right & Turn right & Y \\
\hline & & \multicolumn{1}{c|}{ Percentage } & $80 \%$ \\
\hline
\end{tabular}

As shown in table 4, an experiment of English speech dictation and converting English speech dictation into text found that the English word "back" as a voice command was processed "black". One of the reasons found was due to the non-native speaker's speech accent. The results showed that the English speech command test was $80 \%$ accurate.

With reference to the abovementioned test results, the calculation to determine the accuracy and precision indicated that Thai language voice commands in Table 3 were $100 \%$ accurate and precise, English language voice commands in Table 4 were $80 \%$ accurate and precise, and Thai/English language alternating voice commands in Table 5 were $70 \%$ accurate and precise, and the test results are summarized in Table 6.

Based on the accuracy and precision tests in Table 6 if more homophones in both Thai and English are added, a higher accuracy and precision of the commands can be obtained. 
Table 5. Mobility test result of the automatically moving robot intended for the elderly with Thai/English alternating voice commands.

\begin{tabular}{|c|l|l|c|}
\hline No. & \multicolumn{1}{|c|}{ Voice commands } & \multicolumn{1}{|c|}{ Voice commands to text conversion } & Correctness \\
\hline 1 & Forward $(\mathrm{TH})$ & Forward $(\mathrm{TH})$ & $\mathrm{Y}$ \\
\hline 2 & back $(\mathrm{EN})$ & black $(\mathrm{EN})$ & $\mathrm{N}$ \\
\hline 3 & Turn left $(\mathrm{EN})$ & Turn left $(\mathrm{EN})$ & $\mathrm{Y}$ \\
\hline 4 & Turn right $(\mathrm{EN})$ & Turn right $(\mathrm{EN})$ & $\mathrm{Y}$ \\
\hline 5 & Left $(\mathrm{TH})$ & Left $(\mathrm{TH})$ & $\mathrm{Y}$ \\
\hline 6 & Right $(\mathrm{TH})$ & Right $(\mathrm{TH})$ & $\mathrm{Y}$ \\
\hline 7 & Turn left $(\mathrm{EN})$ & Manicure $(\mathrm{TH})$ & $\mathrm{N}$ \\
\hline 8 & Stop $(\mathrm{EN})$ & Stop $(\mathrm{EN})$ & $\mathrm{Y}$ \\
\hline 9 & Go forward $(\mathrm{EN})$ & Go forward $(\mathrm{EN})$ & $\mathrm{Y}$ \\
\hline 10 & Turn right $(\mathrm{EN})$ & What to do $(\mathrm{TH})$ & $\mathrm{N}$ \\
\hline Percentage & & $70 \%$ \\
\hline
\end{tabular}

As shown in table 5, experiments with Thai/English language switch commands found that every Thai voice language command was accurate. For the English voice command, three voices commands were found that were incorrectly processed: the "back" command is processed into the English word "black", the "turn left" command is processed into a Thai word that means "manicure", and the last command, "turn right", is processed into a Thai word that means "what to do". The experiment results indicated that the accuracy of the language with Thai/English alternating voice commands was $70 \%$.

Table 6. Summary of the mobility test results of the automatically moving robot intended for the elderly with voice control.

\begin{tabular}{|c|l|c|}
\hline \multicolumn{2}{|c|}{ Characteristics in testing } & $\begin{array}{c}\text { Test results in } \\
\text { percentage of accuracy }\end{array}$ \\
\hline 1 & $\begin{array}{l}\text { Mobility of the automatically moving robot intended for the elderly with Thai } \\
\text { language voice commands. }\end{array}$ & $100 \%$ \\
\hline 2 & $\begin{array}{l}\text { Mobility of the automatically moving robot intended for the elderly with } \\
\text { English language voice commands. }\end{array}$ & $80 \%$ \\
\hline 3 & $\begin{array}{l}\text { Mobility of the automotically moving robot intended for the elderly with } \\
\text { Thai/English alternating voice commands. }\end{array}$ & $70 \%$ \\
\hline
\end{tabular}

\section{$5 \quad$ Acknowledgement}

This research is financially supported by the Thailand Research Fund, the TRF Research Team Promotion Grant (RTA), and the Thailand Research Fund under the grant number RTA6280015.

\section{References}

[1] L. Sang et al., "Analysis, Design, and Experimental Research of a Novel WheelchairStretcher Assistive Robot," Appl. Sci., vol. 10, no. 1, 2020, doi: 10.3390/app10010264. 
[2] J. P. Mulhern and A. Stephen J, "Elevated height wheelchair.pdf," 2020.

[3] L. Moody, P. Magee, and D. Stefanov, "Development of a Wheelchair Stability Assessment System: Design Tools and Approaches," in Design of Assistive Technology for Ageing Populations, A. Woodcock, L. Moody, D. McDonagh, A. Jain, and L. C. Jain, Eds. Cham: Springer International Publishing, 2020, pp. 235-256. https://doi.org/10.1007/978-3-03026292-1_13

[4] M. D. P. Justin T. Morgan, Anthony J. Patrick, Joshua J. Jakubowski, Jason Ronald Geidel, "Wheelchair Egress System," 2020.

[5] D. Kumar, R. Malhotra, and S. R. Sharma, "Design and Construction of a Smart Wheelchair," in Procedia Computer Science, 2020, vol. 172, no. 2019, pp. 302-307, doi: 10.1016/j.procs.2020.05.048. https://doi.org/10.1016/j.procs.2020.05.048

[6] P. V Baiju, K. Varghese, J. M. Alapatt, S. J. Joju, and K. M. Sagayam, "Smart Wheelchair for Physically Challenged People," in 2020 6th International Conference on Advanced Computing and Communication Systems (ICACCS), 2020, pp. 828-831, doi: 10.1109/ICACCS48705.2020.9074188. https://doi.org/10.1109/icaccs48705.2020.9074188

[7] B. Sambana, V. S. Patnaik, and N. Thirupathi Rao, "An Artificial Intelligent Approach to User-Friendly Multi-flexible Bed Cum Wheelchair Using Internet of Things," in Smart Technologies in Data Science and Communication, J. Fiaidhi, D. Bhattacharyya, and N. T. Rao, Eds. Singapore: Springer Singapore, 2020, pp. 133-144. https://doi.org/10.1007/978981-15-2407-3_18

[8] A. D. Lacaze, K. N. Murphy, J. Putney, and J. Keyser, "System for automating wheelchair user ingress, egress, and securement on a vehicle," 2020.

[9] D. Sanders et al., "Intelligent Control and HCI for a Powered Wheelchair Using a Simple Expert System and Ultrasonic Sensors," in Intelligent Systems and Applications, K. Arai, S. Kapoor, and R. Bhatia, Eds. Cham: Springer International Publishing, 2021, pp. 571-583. https://doi.org/10.1007/978-3-030-55190-2_42

[10] H. Pan, J. Zhang, and W. Song, "Experimental study of pedestrian flow mixed with wheelchair users through funnel-shaped bottlenecks," J. Stat. Mech. Theory Exp., vol. 2020, no. 3, 2020, doi: https://doi.org/10.1088/1742-5468/ab6b1c

[11] W. Li, J. Talavera, A. G. Samayoa, J. M. Lien, and L. F. Yu, "Automatic Synthesis of Virtual Wheelchair Training Scenarios," in Proceedings - 2020 IEEE Conference on Virtual Reality and 3D User Interfaces, VR 2020, 2020, pp. 539-547, doi:. https://doi.org/10.1109/vr4 $\underline{6266.2020 .1581008046739}$

[12] R. Yaagoubi, Y. Miky, and A. El Shouny, “AccessVOR: A Semantically Enriched VoronoïBased Approach for Navigation Assistance of Wheelchair Users in Indoor Environments," J. Navig., vol. 73, no. 1, pp. 172-191, 2020, doi: https://doi.org/10.1017/s0373463319 00047x

[13] G. Vailland, Y. Gaffary, L. Devigne, V. Gouranton, B. Arnaldi, and M. Babel, "Vestibular Feedback on a Virtual Reality Wheelchair Driving Simulator: A Pilot Study," in Proceedings of the 2020 ACM/IEEE International Conference on Human-Robot Interaction, 2020, pp. 171-179, doi: https://doi.org/10.1145/3319502.3374825

[14] [J. M. Araujo, G. Zhang, J. P. P. Hansen, and S. Puthusserypady, "Exploring Eye-Gaze Wheelchair Control," Eye Track. Res. Appl. Symp., 2020, doi: https://doi.org/10.1145/337 9157.3388933

[15] T. Fearn, F. Labrosse, and P. Shaw, "Wheelchair Navigation: Automatically Adapting to Evolving Environments," in Towards Autonomous Robotic Systems, K. Althoefer, J. Konstantinova, and K. Zhang, Eds. Cham: Springer International Publishing, 2019, pp. 496500. https://doi.org/10.1007/978-3-030-25332-5_49 
[16] S. Niijima, Y. Sasaki, and H. Mizoguchi, "Real-time autonomous navigation of an electric wheelchair in large-scale urban area with 3D map," Adv. Robot., vol. 33, no. 19, pp. 10061018, 2019, doi: https://doi.org/10.1080/01691864.2019.1642240

[17] T. Watanabe, H. Takahashi, Y. Iwasawa, Y. Matsuo, and I. E. Yairi, "Weakly supervised learning for evaluating road surface condition from wheelchair driving data," Inf., vol. 11, no. 1, 2020, doi: https://doi.org/10.3390/info11010002

[18] S. Mascetti, G. Civitarese, O. El Malak, and C. Bettini, "SmartWheels: Detecting urban features for wheelchair users' navigation," Pervasive Mob. Comput., vol. 62, p. 101115, 2020, doi: https://doi.org/10.1016/j.pmcj.2020.101115.

[19] S. Sivakanthan et al., "Usability evaluation of attitude control for a robotic wheelchair for tip mitigation in outdoor environments," Med. Eng. Phys., vol. 82, pp. 86-96, 2020, doi: https://doi.org/10.1016/j.medengphy.2020.07.002

[20] L. Greenberg and W. Stemer, "Standing Wheelchair," 2019.

[21] K. Bayley et al., "Benefits of powered standing wheelchair devices for adolescents with Duchenne muscular dystrophy in the first year of use," J. Paediatr. Child Health, vol. n/a, no. n/a, doi: 10.1111/jpc.14963.

[22] S. Jeong, N. Maeda, and K. Kozai, "Driving Assist Control of Wheeled Inverted Pendulum Wheelchair Using an Active Seat-Slider," in 2019 19th International Conference on Control, Automation and Systems (ICCAS), 2019, pp. 162-167, doi: https://doi.org/10.2391 9/iccas47443.2019.8971752

[23] M. K. Shahin, A. Tharwat, T. Gaber, and A. E. Hassanien, "A Wheelchair Control System Using Human-Machine Interaction: Single-Modal and Multimodal Approaches," J. Intell. Syst., vol. 28, no. 1, pp. 115-132, 2019, doi: https://doi.org/10.1515/jisys-2017-0085

[24] M. Xiong et al., "A Low-Cost, Semi-Autonomous Wheelchair Controlled by Motor Imagery and Jaw Muscle Activation," in 2019 IEEE International Conference on Systems, Man and Cybernetics (SMC), 2019, pp. 2180-2185, doi: https://doi.org/10.1109/smc.2019.8914415

[25] K. Li, S. Ramkumar, J. Thimmiaraja, and S. Diwakaran, "Optimized artificial neural network based performance analysis of wheelchair movement for ALS patients," Artif. Intell. Med., vol. 102, no. November 2019, 2020, doi: https://doi.org/10.1016/j.artmed.20 $\underline{19.101754}$

[26] M. Alabboudi, M. Majed, F. Hassan, and A. B. Nassif, "EEG wheelchair for people of determination," 2020 Adv. Sci. Eng. Technol. Int. Conf. ASET 2020, 2020, doi: https://doi.org/10.1109/aset48392.2020.9118340

[27] M. A. Awais, M. Z. Yusoff, N. Yahya, S. Z. Ahmed, and M. U. Qamar, "Brain Controlled Wheelchair: A Smart Prototype," J. Phys. Conf. Ser., vol. 1529, no. 4, 2020, doi: https://doi.org/10.1088/1742-6596/1529/4/042075

[28] H. M. R. T. Bandara, K. S. Priyanayana, A. G. B. P. Jayasekara, D. P. Chandima, and R. A. R. C. Gopura, "An Intelligent Gesture Classification Model for Domestic Wheelchair Navigation with Gesture Variance Compensation," Appl. Bionics Biomech., vol. 2020, p. 9160528, 2020, doi: https://doi.org/10.1155/2020/9160528

[29] A. S. Kundu, O. Mazumder, P. K. Lenka, and S. Bhaumik, "Hand Gesture Recognition Based Omnidirectional Wheelchair Control Using IMU and EMG Sensors," J. Intell. Robot. Syst., vol. 91, no. 3, pp. 529-541, 2018, doi: https://doi.org/10.1007/s10846-017-0725-0

[30] M. Dahmani et al., "An intelligent and low-cost eye-tracking system for motorized wheelchair control," Sensors (Switzerland), vol. 20, no. 14, pp. 1-27, 2020, doi: https://doi.org/10.3390/s20143936

[31] G. Caiza, C. Reinoso, H. Vallejo, M. Albarracín, and E. P. Salazar, "Semi-automatic Eye Movement-Controlled Wheelchair Using Low-Cost Embedded System," in Trends and 
Innovations in Information Systems and Technologies, 2020, pp. 755-764. https://doi.org/ 10.1007/978-3-030-45691-7_71

[32] A. A. Q. Mohd, M. F. M. Khairudin, L. H. Basirun, and R. Jailani, "Motorised Wheelchair with Multicontrol System," in ISCAIE 2020 - IEEE 10th Symposium on Computer Applications and Industrial Electronics, 2020, pp. 233-238, doi: https://doi.org/10.1109/ iscaie47305.2020.9108831

[33] N. Herron et al., "Wheelchair safety systems and related methods," 2020.

[34] D. A. Moore, "Autonomously moveable storage units and wheelchair storage systems," 2020.

[35] B. Champaty, S. K. Nayak, G. Thakur, B. Mohapatra, D. N. Tibarewala, and K. Pal, Development of Bluetooth, Xbee, and Wi-Fi-Based Wireless Control Systems for Controlling Electric-Powered Robotic Vehicle Wheelchair Prototype, no. June. 2016. https://doi.org/10. 4018/978-1-7998-1754-3.ch052

[36] C. Mohan and H. K. Verma, "Futuristic integrated assistive system for elderly population of India," Int. J. online Biomed. Eng., vol. 15, no. 13, pp. 34-45, 2019, doi: https://doi.org/ 10.3991/ijoe.v15i13.11058

[37] J. S. Wilson, Ed., "Front Matter," in Sensor Technology Handbook, Burlington: Newnes, 2005, p. iii.

[38] F. Tian, X. Long, and W. Liao, "Design of smart home system based on basic radio frequency wireless sensor network," Int. J. Online Eng., vol. 14, no. 4, pp. 126-136, 2018, doi: https://doi.org/10.3991/ijoe.v14i04.8389

[39] D. J. Brems and M. S. Schoeffler, “Automatic speech recognition (ASR) processing using confidence measures," 1997.

[40] B. M. Ballinger, J. Schalkwyk, M. H. Cohen, and C. G. Luc Allauzen, "Language model selection for speech-to-text conversion," 2010.

[41] J. Li, L. Deng, R. Haeb-Umbach, and Y. Gong, Fundamentals of speech recognition. 2016.

[42] J. McKenna, "Speech to text mothod and system," 2002.

[43] N. Shakhovska, O. Basystiuk, and K. Shakhovska, "Development of the speech-to-text chatbot interface based on google API," CEUR Workshop Proc., vol. 2386, pp. 212-221, 2019.

[44] M. Hinderer, P. Friedrich, and B. Wolf, “An autonomous stair-climbing wheelchair," Rob. Auton. Syst., vol. 94, pp. 219-225, 2017, doi: https://doi.org/10.1016/j.robot.2017.04.015

[45] S. Hashizume, I. Suzuki, K. Takazawa, R. Sasaki, and Y. Ochiai, "Telewheelchair: The remote controllable electric wheelchair system combined human and machine intelligence," ACM Int. Conf. Proceeding Ser., 2018, doi: https://doi.org/10.1145/3174910.3174914

[46] R. Josephine Leela, A. Joshi, B. Agasthiya, U. K. Aarthiee, E. Jameela, and S. Varshitha, "Android based automated wheelchair control," in Proceedings - 2017 2nd International Conference on Recent Trends and Challenges in Computational Models, ICRTCCM 2017, 2017, pp. 349-353, doi: https://doi.org/10.1109/icrtccm.2017.44

[47] N. Aktar, I. Jaharr, and B. Lala, "Voice Recognition based intelligent Wheelchair and GPS Tracking System," in 2nd International Conference on Electrical, Computer and Communication Engineering, ECCE 2019, 2019, pp. 7-9, doi:. https://doi.org/10.1109/ ecace. 2019.8679163

[48] S. R. Avutu, D. Bhatia, and B. Venkateswara Reddy, "Voice control module for low cost local-map navigation based intelligent wheelchair," in Proceedings - 7th IEEE International Advanced Computing Conference, IACC 2017, 2017, pp. 609-613, doi: https://doi.org/10. $\underline{1109 / \text { iacc.2017.0129 }}$ 
[49] J. Barbosa, J. Tavares, I. Cardoso, B. Alves, and B. Martini, "TrailCare: An indoor and outdoor Context-aware system to assist wheelchair users," Int. J. Hum. Comput. Stud., vol. 116, no. April, pp. 1-14, 2018, doi: https://doi.org/10.1016/j.ijhcs.2018.04.001

[50] D. Puanhvuan, S. Khemmachotikun, P. Wechakarn, B. Wijarn, and Y. Wongsawat, "Navigation-synchronized multimodal control wheelchair from brain to alternative assistive technologies for persons with severe disabilities," Cogn. Neurodyn., vol. 11, no. 2, pp. $117-$ 134, 2017, doi: https://doi.org/10.1007/s11571-017-9424-6

[51] A. S. Kundu, O. Mazumder, P. K. Lenka, and S. Bhaumik, "Hand Gesture Recognition Based Omnidirectional Wheelchair Control Using IMU and EMG Sensors," J. Intell. Robot. Syst. Theory Appl., vol. 91, no. 3-4, pp. 529-541, 2018, doi: https://doi.org/10.1007/s10846$\underline{017-0725-0}$

[52] L. Coelho and D. Braga, "ezGo: A Voice Operated Wheelchair with Biosignal Monitoring for Home Environments," Dsai 2009 Proc. 2nd Int. Conf. Softw. Dev. Enhancing Access. Fight. Info-Exclusion, no. February 2014, pp. 125-130, 2009.

[53] S. Shaheen and A. Umamakeswari, "Intelligent wheelchair for people with disabilities," Int. J. Eng. Technol., vol. 5, no. 1, pp. 391-397, 2013.

[54] B. G. Kumar and K. Sudhagar, "Development of stair climbing intelligent wheelchair for physically disabled people," vol. 9, pp. 2717-2725, 2016.

[55] E. Yulianto, T. B. Indrato., B. TMN, and Suharyati, "Wheelchair for Quadriplegic Patient with Electromyography Signal Control Wireless," Int. J. online Biomed. Eng., vol. 16, no. 12, pp. 94-115, 2020, doi: https://doi.org/10.3991/ijoe.v16i12.15721

\section{$7 \quad$ Authors}

Manussawee Piyaneeranart received the B.E. degree in Computer Science from the Suan Dusit University, Thailand, in 2000, and the MS.I.Ed. Computer Technology, King Mongkut's University of Technology North Bangkok, Thailand, in 2006 and the study Ph.D. degrees in Information Technology from Information Technology and Digital Innovation, King Mongkut's University of Technology North Bangkok, Thailand. She is currently a government officer, Thailand s5907011910045@email.kmut nb.ac.th .

Mahasak Ketcham received the B.B.A. in Business Computer Siam University, Thailand, MS.I.Ed. Computer Technology, King Mongkut's University of Technology North Bangkok, Thailand and Ph.D. in Computer Engineering, Chulalongkorn University, Thailand. Dr. Mahasak Ketcham is an assistant professor in Department of Information Technology Management, King Mongkut's University of Technology North Bangkok, Thailand (email: mahasak.k@itd.kmutnb.ac.th).

Article submitted 2021-02-24. Resubmitted 2021-03-16. Final acceptance 2021-03-18. Final version published as submitted by the authors. 